\title{
APLICACIÓN DEL TEST COURSE NAVETTE EN ESCOLARES
}

COURSE NAVETTE TEST APLICACTION IN SCHOOL

\begin{abstract}
CURILEM GATICA, Cristian; ALMAGIÀ FLORES, Atilio \& YUING FARÍAS, Tuillang.
Laboratorio de Antropología Física y Anatomía Humana, Instituto de Biología, Facultad de Ciencias, Pontificia Universidad Católica de Valparaíso. Chile.
\end{abstract}

CURILEM GATICA, C.; ALMAGIÀ FLORES, A. \& YUING FARÍAS, T. (2015). Aplicación del Test Course Navette en escolares. Mot. Hum. 16(2); 95-99.

\section{RESUMEN}

El desarrollo de la resistencia cardiorrespiratoria en niños, adolescentes y jóvenes se asocia a la disminución de la prevalencia de enfermedades cardiovasculares en niños y adolescentes. La prueba de "course navette" o carrera de 20 metros es una prueba de aptitud cardiorrespiratoria máxima y progresiva, que mide indirectamente el consumo máximo de oxigeno. Niveles altos de capacidad aeróbica durante la niñez y la adolescencia están asociados con una salud cardiovascular actual y futura más saludable. Según la encuesta nacional de salud en Chile (2009-2010) el 17,7\% de la población chilena mayor de 15 años y más, presenta un riesgo cardiovascular alto o muy alto. La población chilena presenta: un elevado nivel de morbilidad por enfermedades crónicas no transmisibles y de sus factores de riesgo. Utilizar una prueba máxima y progresiva en adolescentes, como es la "prueba de course naveta", puede genera un aumento del riesgo de presentar un evento cardiovascular al evaluar el consumo máximo de oxigeno en nuestra población.

Palabras Clave: Consumo máximo de oxigeno, adolescentes, condición física.

\begin{abstract}
The development of cardiorespiratory endurance in children and young people is associated with decreased prevalence of cardiovascular disease in children and adolescents. The test of "course navette" or 20 meter race is a test of high cardiorespiratory fitness and progressive, which indirectly measures the maximum oxygen consumption. High levels of aerobic fitness during childhood and adolescence are associated with a current cardiovascular health and healthier future. According to the health survey in Chile, $17.7 \%$ of the Chilean population 15 years and over, has a high or very high cardiovascular risk. The Chilean population has: a high level of morbidity from chronic diseases and their risk factors of disease. Use a high and progressive test in adolescents, such as "proof of course navetta" can generate an increased risk of cardiovascular events in assessing the maximum oxygen consumption in our

population.
\end{abstract}

Keywords: maximum oxygen consumption, teen, fitness. 


\section{INTRODUCCIÓN}

El termino condición física ha ido evolucionando a lo largo del tiempo, pasando de una orientación tradicional vinculada al rendimiento deportivo hacia una orientación mucho más cercana y relacionada con la salud. En general, se entiende por resistencia cardiorrespiratoria (RCR) a la capacidad para realizar tareas moderadas que impliquen la participación de grandes masas musculares durante periodos prolongados de tiempo. El desarrollo de la RCR en niños, adolescentes y jóvenes se asocia a la disminución de la prevalencia de enfermedades cardiovasculares en niños y adolescentes. Además, una alta capacidad cardiorrespiratoria durante la infancia y la adolescencia se asocia con un perfil cardiovascular más saludable tanto en la niñez como en la vida adulta (Mayorga-Vega, Brenes, Rodriguez, \& Merino, 2012; López et al., 2013).

Las enfermedades cardiovasculares tienen su inicio en la infancia y los factores de riesgo para la enfermedad cardiovascular y enfermedad metabólica, como son la obesidad abdominal, la tensión arterial elevada, la resistencia a la insulina y los triglicéridos elevados, son observables en niños $\mathrm{y}$ adolescentes y tienden a persistir desde la infancia hasta la vida adulta (Kain et al., 2004; MINSAL, 2010). Por lo tanto, la evaluación de la condición física relacionada con la salud en estas edades es de gran interés desde el ámbito clínico y de la salud pública. La condición física relacionada con la salud incluye como principales componentes la capacidad aeróbica, capacidad musculo-esquelética, capacidad motora y composición corporal. Niveles altos de capacidad aeróbica durante la niñez y la adolescencia están asociados con una salud cardiovascular actual y futura más saludable (Aránguiz et al., 2010; Assessing levels of physical activity, 2011).

\section{Test Course Navette}

La prueba de "course naveta" o carrera de 20 metros es una prueba de aptitud cardiorrespiratoria máxima y progresiva, que mide la potencia aeróbica máxima e indirectamente el consumo máximo de oxigeno, se realiza mediante una carrera de ida $(20$ metros) y vuelta (20 metros) en la que el sujeto va desplazándose de un punto a otro, realizando un cambio del sentido al ritmo indicado por una señal sonora que va acelerándose progresivamente. La prueba se inicia a una velocidad de $8 \mathrm{~km} / \mathrm{h}$, siendo al principio lento, incrementándose la velocidad paulatinamente con el transcurso del tiempo, la prueba culmina a una velocidad de $18 \mathrm{~km} / \mathrm{h}$. Durante la prueba, el mismo sujeto es el encargado de determinar el propio ritmo, de tal manera que se encuentre en un extremo de la pista al oír la señal, con una aproximación de 1 ó 2 metros. En cada extremo hay que tocar la línea con el pie y la prueba acaba en el momento en que el individuo interrumpe voluntariamente la prueba, y/o cuando no soportan más el ritmo impuesto por la señal acústica (Torres-Luque, Carpio, Lara, \& Zagalaz, 2014; Gomez-Campos et al., 2014).

\section{Viabilidad de su aplicación}

El consumo máximo de oxigeno se expresa en litros por minutos o en mililitros por kilogramo por minuto, para valorar la condición física del sujeto expresado en valores relativos. Cuanto mayor sea este valor, mayor capacidad tendrá ese organismo para producir energía mediante el metabolismo aeróbico, menor necesidad de recurrir al metabolismo anaeróbico láctico y mayor capacidad de eliminación de acido láctico en caso de haber sido producido (Corral Pernia \& del Catillo, 2010).

Los niveles de condición física están determinados por un conjunto de factores, siendo los factores genéticos, que se manifiestan básicamente en los procesos de crecimiento, desarrollo y maduración, responsables del $40 \%$ ó más de la variación total de la condición física. Otro factor importante es el factor ambiental que incluye la actividad física, entendida como cualquier movimiento corporal producido por los músculos esqueléticos que resulte en gasto calórico, y el ejercicio físico, considerado como la actividad física planificada, estructurada, sistematizada y deliberada. Las pruebas que valoran la resistencia aeróbica están influenciadas por factores motivacionales y por aspectos relacionados con las dimensiones corporales (Fajardo \& Ángel, 2012; Bustamante, Beunen, \& Maia, 2012). 
Las clases de educación física requieren aumentar la intensidad para producir mejoras en la capacidad aeróbica de los adolescentes, individualizar la intensidad del ejercicio en función de la capacidad de cada estudiante y controlar la intensidad prescrita durante las clases, son necesarias intensidades mayores al $80 \%$ de la frecuencia cardiaca máxima para producir mejoras significativas en el consumo máximo de oxigeno (Vargas, 2012; Ramírez et al., 2012).

Los niños no son adultos pequeños, su fisiología es diferente; estos crecen, y como tal, las respuestas fisiológicas y metabólicas al esfuerzo varían de acuerdo y a medida que se desarrollan a lo largo de la infancia y la adolescencia. Con relación al perfil bioenergético los niños tienen menor capacidad anaeróbica o glucolítica para producir adenosís trifosfato durante el ejercicio físico. Los niños tienen diferente respuesta cardiovascular, ventilatorias y metabólicas durante la prueba de esfuerzo progresivo máximo en comparación a los adultos: un valor mayor de la frecuencia cardiaca en la misma carga a la que son sometidos los adultos, este es un mecanismo compensatorio para un menor volumen cardiaco y menor volumen sistólico, relacionados con un menor tamaño del corazón, menor volumen sistólico, menor masa muscular resultando en retorno venoso atenuado (precarga) en niños. Los niños presentan alteraciones en las respuestas ventilatoria durante el esfuerzo progresivo, muestran menor eficiencia con valores mayores de frecuencia ventilatoria y menores valores de volumen corriente durante la prueba de esfuerzo. El patrón ventilatorio en la población pediátrica depende del estado de la madurez, se observa una ventilación excesiva durante el esfuerzo físico. Los niños tienen menor capacidad glucolítica que los adultos, esta inmadurez en el metabolismo anaeróbico en niños observada durante el ejercicio físico presenta diversas razones: menor nivel de glucógeno muscular, actividad reducida de fosfofructoquinasa-1 y lactato deshidrogenasa y mayor proporción de fibras de contracción lenta (Galaviz et al., 2012; Oviedo et al., 2013; Leite et al., 2010).
Un meta-análisis reciente hecho en 57 investigaciones define que la validez de criterio del protocolo fue estadísticamente superior para adultos $(\mathrm{RP}=0.94,0.87-1.00)$ que para los niños $(\mathrm{RP}=$ $0,78,0,72-0,85)$. En adultos, la puntuación de rendimiento sólo parece ser un fuerte estimador de la capacidad cardiorrespiratoria, en contraste entre los niños de la puntuación de los resultados debe combinarse con otras variables (sexo, peso y el IMC), para mejorar la estimación, además de ser simplemente una estimación y no una medida directa de la capacidad cardiorrespiratoria ni del VO2max (Mayorga-Vega, Aguilar-Soto \& Viciana, 2015).

Un estudio de Batista et al (2013) indican una amplia variación en la precisión y la concordancia entre las ecuaciones utilizadas en la estimación del VO2max. Por lo tanto, nuestros resultados sugieren que las ecuaciones de Barnett et al. (1993), Leger et al. (1992), Mahar et al. (2006), y Matsuzaka et al. (2004) son inadecuados para evaluar la capacidad cardiorrespiratoria en test de $20 \mathrm{~m}$ en adolescentes de 11-13 años.

\section{CONCLUSIONES}

Al presentar nuestra población sedentaria un riesgo cardiovascular de $48 \%$ en hombres y $54 \%$ en mujeres (Celis-Morales et al., 2015), un sobrepeso evidente y un nivel de sedentarismo alto. Utilizar una prueba máxima y progresiva en adolescentes, como es la "prueba de navetta", genera un aumento del riesgo de presentar un evento cardiovascular al evaluar el consumo máximo de oxigeno. Existen pruebas con metodología submaximal que pueden ser implementadas en el ámbito escolar (Gatica et al., 2012), que pueden evitar problemas de salud cardiovascular para nuestra población infantojuvenil, además de ser mas efectivas para el desarrollo de los joevenes escolares. Es necesario utilizar y validar pruebas para evaluar la condición física en nuestra población, tomando en cuenta los niveles de obesidad, sedentarismo y factores propios de los adolescentes chilenos. 


\section{AGRADECIMIENTOS}

En primer lugar a Jesucristo nuestro Redentor, a cada una de las personas participantes de la investigación, al Jefe Docente e Investigador del Laboratorio de Antropología Física y Anatomía Humana de la Pontificia Universidad Católica de Valparaíso, por la constancia y colaboración en el trabajo. A Fernando Rodríguez. Por otro lado agradecer a la Dirección de Investigación, Vicerectoría de Investigación y Estudios Avanzados e Instituto de Biología de la Facultad de Ciencias. Pontificia Universidad Católica de Valparaíso. Chile, por su constante apoyo en materia investigativa.

\section{REFERENCIAS BIBLIOGRÁFICAS}

1. Aránguiz, H., García, V., Rojas, S., Salas, C., Martínez, R., \& MacMillan, N. (2010). Estudio descriptivo, comparativo $\mathrm{y}$ correlacional del estado nutricional $\mathrm{y}$ condición cardiorrespiratoria en estudiantes universitarios de Chile. Revista Chilena de Nutrición, 70-79.

2. Assessing levels of physical activity. (2011). Batería ALPHA-fitness: test de campo para la evaluación de la condición física relacionada con la salud en niños $y$ adolescentes. Nutrición hospitalaria, 12101214.

3. Barnett, A, Chan, LYS, \& Bruce, IC. (1993). A preliminary study of the $20-\mathrm{m}$ multistage shuttle run as a predictor of peak VO2 in Hong Kong Chinese students. Pediatr Exerc Sci 5: 42-50.

4. Batista, M. B., Cyrino, E. S., Arruda, M., Dourado, A. C., Coelho-E-Silva, M. J., Ohara, D., ... \& Ronque, E. R. (2013). Validity of Equations for Estimating $\mathrm{V}$ [Combining Dot Above] O2peak From the 20-m Shuttle Run Test in Adolescents Aged 11-13 Years. The Journal of Strength \& Conditioning Research, 27(10), 2774-2781.

5. Bustamante, A., Beunen, G., \& Maia, J. (2012). Valoración de la aptitud física en niños y adolescentes: construcción de cartas percentílicas para la región central de Perú. Revista Perú med exp salud pública, 188197.

6. Celis-Morales, Carlos, Salas, Carlos, Álvarez, Cristian, Aguilar Farías, Nicolás, Ramírez Campillos, Rodrigo, Leppe, Jaime, Cristi-Montero, Carlos, Díaz Martínez, Ximena, Duran, Eliana, Labraña, Ana María, Martínez, María Adela, Leiva, Ana María, \& Willis, Naomi. (2015). Higher physical activity levels are associated with lower prevalence of cardiovascular risk factors in Chile. Revista médica de Chile, 143(11), 1435-1443.

7. Corral Pernia, J., \& del Catillo, O. (2010). La valoración del VO2 máx. y su relación con el riesgo cardiovascular como medio de enseñanza-aprendizaje. Cuadernos de psicología del deporte, 25-30.

8. Fajardo, E., \& Ángel, L. (2012). Prevalencia de sobrepeso y obesidad, consumo de alimentos y patrón de actividad física en una población de niños escolares de la ciudad de Bogotá. Revista facultad medicina, 101-116.

9. Galaviz, K., Tremblay, M., Colley, R., Jáuregui, E., López, J., \& Janssen, I. (2012). Associations between physical activity, cardiorespiratory fitness, and obesity in Mexican children. Salud pública de México, 463-470.

10. Gatica, D., Puppo, H., Villaroel, G., San Martin, I., Lagos, R., Montecinos, J., y otros. (2012). Valores de referencia del test de marcha de seis minutos en niños sanos. Revsita Med Chile, 1014-1021.

11. MINSAL (2010). Encuesta nacional de salud. Santiago: Ministerio de salud.

12. Gomez-Campos, R., Arruda, M., Almonacid-Fierro, A., Holbold, E., AmaralCamargo, C., Gamero, D., y otros. (2014). Capacidad cardiorrespiratoria de niños escolares que viven a moderada altitud. Revista Chilena de pediatría, 188-196.

13. Kain, J., Olivares, S., Romo, M., Leyton, B., Vio, F., Cerda, R., y otros. (2004). Estado 
nutricional y resistencia aeróbica en escolares de educación básica: línea base de un proyecto de promoción de la salud. Revista Médica de Chile, 1395-1402.

14. Le' ger, LA, Mercier, D, Gadoury, C, and Lambert, J. (1988). The multistage 20-meter shuttle run test for aerobic fitness. J Sports Sci 6: 93-101.

15. Leite, D., Wanderley, A., Pinto, M., Ferreira, L., Matos, L., Negrao, C., y otros. (2010). Comportamiento cardiorrespiratorio en niños saludables durante el ejercicio progresivo máximo. Arq Bras Cardiol, 477483.

16. López-Jaramillo, P., Gómez-Arbeláez, D., Cohen, D., Camacho, P., Rincón-Romero, K., Hormiga, C., y otros. (2013). Asociación entre obesidad y baja capacidad muscular y función cardiorrespiratoria, factores de riesgo cardiometabólico en niños colombianos. Trauma fundación Mapfre, 1723.

17. Mahar, MT, Welk, GJ, Rowe, DA, Crotts, DJ, and McIver, KL. (2006). Development and validation of a regression model to estimate VO2peak from PACER 20-m shuttle run performance. J Phys Act Health 3: S34-S46.

18. Matsuzaka, A, Takahashi, Y, Yamazoe, M, Kumakura, N, Ikeda, A, Wilk, B, and BarOr, O. (2004). Validity of the multistage 20$\mathrm{m}$ shuttle-run test for Japanese children, adolescents, and adults. Pediatr Exerc Sci 16: 113-125.

19. Mayorga-Vega, D., Brenes, A., Rodriguez, M., \& Merino, R. (2012). Asociación entre IMC y el nivel de condición física en escolares de educación primaría. Journal of sport and health research, 299-310.

20. Mayorga-Vega D., Aguilar-Soto P. \& Viciana J. (2015). Criterion-Related Validity of the 20-M Shuttle Run Test for Estimating Cardi- orespiratory Fitness: A MetaAnalysis. Journal of Sports Science and Medicine 14, 536-547.
21. Oviedo, G., Sánchez, J., Castro, R., Calvo, M., Sevilla, J., Iglesias, A., y otros. (2013). Niveles de actividad física en población adolescente: estudio de caso. Retos: nuevas tendencias en educación física, deporte $\mathrm{y}$ recreación, 43-47.

22. Ramírez, J., Muros, J., Morente, J., Sánchez, C., Femia, P., \& Zabala, M. (2012). Efecto de un programa de entrenamiento aeróbico de 8 semanas durante las clases de educación física en adolescentes. Nutrición hospitalaría, 747-754.

23. Torres-Luque, G., Carpio, E., Lara, A., \& Zagalaz, M. (2014). Niveles de condición física de escolares de educación primaria en relación a su nivel de actividad física y al género. Retos: nuevas tendencias en educación física, deporte y recreación, 1722.

24. Vargas, M. (2012). La medición de la actividad física diaria como alternativa al test de ejercicio en la evaluación preoperatoria del riesgo en la resección pulmonar. Salamanca: Universidad de Salamanca.

\section{Dirigir correspondencia a::}

Cristian Arturo Curilem Gatica

Laboratorio de Antropología Física y Anatomía

Humana

Instituto de Biología, Facultad de Ciencias

Avenida Universidad 330, Curauma, Placilla

Pontificia Universidad Católica de Valparaíso

CHILE

Email: c.curilem.g@gmail.com

\section{RECIBIDO: 1-12-2015 \\ ACEPTADO: 29-12-2015}

